\title{
Propagation des ondes électromagnétiques en présence d'un matériau ferromagnétique
}

\author{
Jean-Luc Joly * \\ Guy Métivier ${ }^{\dagger}$ \\ et \\ Jeffrey Rauch ${ }^{\ddagger}$
}

\section{Résumé}

Les textes physiques fondateurs de Bloch, Landau et Lifshitz et Néel sur le ferromagnétisme remontent aux années 30 et la recherche physique dans ce domaine est active. Les travaux mathématiques et numériques sur le sujet sont en revanche encore peu nombreux et récents. Ils laissent cependant deviner la richesse du domaine tant du point de vue théorique et numérique que de celui des applications. Un point même imparfait a semblé utile.

Research partially supported by

the U.S. National Science Foundation, U.S. Office of Naval Research, and the NSF-CNRS cooperation program under grants number

NSF-DMS-9203413 and OD-G-N0014-92-J-1245 NSF-INT-9314095 respectively, and the CNRS through the Groupe de Recherche G1180 POAN.

\section{Présentation du modèle}

La littérature physique sur le sujet est abondante. Les articles de Bloch [5], Landau et Lifshitz [24] et Néel [28] sont les contributions fondatrices qui datent des années 30 et 40. Le domaine connait un nouveau développement dans les années 60 avec la naissance de l'informatique moderne et le besoin de stockage de l'information sur des supports magnétiques, plus récemment à cause de l'intérêt pour des matériaux magnétiques susceptibles d'absorber en partie les ondes électromagnétiques. Un des buts du sujet est l'étude de la structuration du matériau ferromagnétique en domaines séparés par des parois ou murs (murs de Bloch, de Néel) qui sont des zones où la magnétisation du matériau change rapidement de direction. Ce phénomène devient apparent lorsque certains paramètres sont très petits. À la limite, ces parois pourraient apparaître comme le support singulier de la magnétisation. Pour une première approche on peut consulter l'exposé de Miltat [27] avec sa bibliographie ainsi que le livre de Hubert [17].

Les résultats mathématiques sur le sujet sont encore peu nombreux et récents. L'intérêt du sujet réside dans ses connections avec des domaines variés et différents des équations aux

*MAB, Université Bordeaux I, 33405 Talence, FRANCE.

†TMAR, Université Rennes I, 35042 Rennes, FRANCE.

${ }_{\ddagger}^{\ddagger}$ Department of Mathematics, University of Michigan, Ann Arbor 48109 MI, USA. 
dérivées partielles - applications harmoniques, équations hyperboliques notamment - et dans le fait qu'il pose de nombreuses questions, numériques et théoriques, certaines sans doute difficiles. Sans prétention à l'exhaustivité, cet exposé tente de faire un point plus incitatif que précis sur les aspects mathématiques de la question avec un peu plus de détails sur un de ses aspects étudié par les auteurs de ce texte.

D'abord quelques mots sur le modèle. On se limite à une version très simplifiée où le corps ferromagnétique est supposé isotrope et où il n'y a pas de charges électriques, où finalement ne sont prises en compte que l'énergie d'échange et l'énergie démagnétisante. L'induction et le champ électriques $D$ et $E$ définis dans $\mathbb{R}^{3}$ satisfont

$$
D=\varepsilon_{0} E,
$$

où $\varepsilon_{0}$ est un scalaire positif. L’induction magnétique vérifie

$$
\left.B(t, x)=\mu_{0} H(t, x)+4 \pi M(t, x)\right), x \in \Omega, \quad B(t, x)=\mu_{0} H(t, x), x \in \mathbb{R}^{3} \backslash \bar{\Omega}
$$

où $H$ est le champ magnétique, $\mu_{0}$ un coefficient positif et $M$ la magnétisation du milieu ferromagnétique qui occupe la partie de l'espace définie par $\Omega$, un ensemble ouvert et régulier de $\mathbb{R}^{3}$. On peut choisir un système d'unités tel que $\varepsilon_{0}=\mu_{0}=1$, donc tel que la vitesse de la lumière $c=1$ et les facteurs $4 \pi$ disparaissent. On suppose que $M$, comme $E$ et $H$, est une distribution sur $\mathbb{R}^{3}$ à valeurs dans $\mathbb{R}^{3}, M$ étant nulle sur $\mathbb{R}^{3} \backslash \bar{\Omega}$ en vertu de (1).

S'agissant des équations on a d'abord le système de Maxwell

$$
\left\{\begin{array}{l}
\partial_{t} D-\operatorname{rot} H=0, \quad t \geq 0, x \in \mathbb{R}^{3} \\
\partial_{t} B+\operatorname{rot} E=0 \quad t \geq 0, x \in \mathbb{R}^{3},
\end{array}\right.
$$

tandis que la magnétisation obéit à l'équation dite de Landau-Lifschitz qui s'écrit

$$
\partial_{t} M=F\left(M, H+H_{e}\right), t \geq 0, x \in \mathbb{R}^{3} .
$$

Dans (3) on doit préciser ce que représentent $F$ et $H_{e}$. D'abord $F$, le terme d'interaction non linéaire. Il a la forme suivante

$$
F(m, h):=\frac{\gamma}{1+\alpha^{2}}\left(m \wedge h-\frac{\alpha}{|m|}(m \wedge(m \wedge h))\right), \quad h, m \in \mathbb{R}^{3},
$$

où $\gamma$, la constante gyromagnetique, et $\alpha$, qui représente un coefficient d'amortissement, sont tous deux positifs.

Ensuite $H_{e}$, le champ effectif, dépend de $M$ et même des dérivées de $M$ et s'écrit

$$
H_{e}=\varepsilon \mathcal{I}_{\mid \Omega} \triangle M
$$

avec $\varepsilon \geq 0$. Ce terme $H_{e}$, dit terme d'échange, dérive d'une énergie (d'échange),

$$
\mathcal{E}_{e}(M)=\varepsilon \int_{\Omega}|\nabla M|^{2},
$$

où $|\nabla M|^{2}=\sum_{1<i, j<3}\left|\partial_{i} M_{j}\right|^{2}$. Des modèles plus complets prennent en compte des relations entre les propriétés physiques et magnétiques du matériau (énergie d'anisotropie, etc.). On trouvera dans [3] et [21], [22] des résultats mathématiques où ces termes sont considérés.

Dans le cas présent où il n'y a pas de charges électriques on a

$$
\operatorname{div} E=0 \text {. }
$$

Le principe de Faraday, qui énonce qu'il n’y a pas de charges magnétiques, donne

$$
\operatorname{div} B=0 .
$$


Les deux conditions sur la divergence sont propagées dans le temps par les équations de Maxwell: si on s'intéresse au problème de Cauchy elles sont vérifiées partout dès qu'elles le sont à l'instant initial $t=0$.

La nouveauté par rapport au système de Maxwell dans le vide est le couplage avec l'équation de Landau-Lifshitz qui est une équation différentielle non linéaire si $\varepsilon=0$ et une équation aux dérivées partielle non linéaire dans le cas contraire. Dans ce dernier cas il faut rajouter à (3) une condition de transmission sur le bord de $\Omega$ qui s'écrit

$$
\partial_{n} M_{\mid \partial \Omega} \wedge M_{\mid \partial \Omega}=0 .
$$

La propriété fondamentale que présente l'équation de Landau-Lifshitz est la conservation de la longueur du vecteur $M(t, x)$ au cours du temps qui résulte de l'observation que

$$
F(m, h) \cdot m=0
$$

et qui entraîne (au moins formellement si $\varepsilon \neq 0$ ), après multiplication de $(3)$ par $M(t, x)$, que

$$
\frac{1}{2} \partial_{t}|M(t, x)|^{2}=0 .
$$

Arrêtons nous un instant sur la dynamique définie par (3) en supposant que $H$ est un vecteur fixe. Examinons d'abord la situation où $\varepsilon=0$. Alors, si $\alpha=0$, le mouvement de $M$ est un mouvement de rotation positive et uniforme autour de l'axe $-H$ de fréquence $\gamma|H|$. Si $\alpha>0$, $M$ tourne toujours dans le sens positif autour de $-H$ sur une sphère centrée à l'origine et avec une fréquence $\frac{\gamma}{1+\alpha^{2}}$ indépendant de $|M|$ mais tend à s'aligner avec $H$ quand $t \rightarrow \infty$. Considérons maintenant le cas $\varepsilon=1$ et $H=0$. Alouges et Soyeur [4] ont montré l'existence de solutions faibles et prouvé que pour certaines conditions initiales il y en avait même une infinité. Lorsque $\alpha \rightarrow 0$ les solutions convergent vers des solutions de l'équation de Heisenberg

$$
\partial_{t} M=M \wedge \triangle M .
$$

On constate donc que le laplacien n'est pas aussi régularisant qu'on pourrait le penser d'abord et que la dynamique présente une forme d'instabilité. En tout cas mathématiquement les deux cas $\varepsilon=0$ et $\varepsilon \neq 0$ sont bien différents.

La section 2 donne un aperçu très succint des problèmes et résultats lorsque $\varepsilon \neq 0$. Le paragraphe 3 présente quelques énoncés sur le problème de Cauchy dans le cas $\varepsilon=0$. La suite de l'exposé donne quelques détails techniques sur la démonstration de ces derniers énoncés.

Comme on le verra les résultats partiels déjà obtenus indiquent que les problèmes possèdent en général des solutions à basse régularité (celles qui sont naturellement associées aux énergies), qui peuvent même être stables dans les topologies considérées. Cependant tous les problèmes semblent manifester une certaine instabilité sur les questions d'unicité et de régularité. Concernant l'unicité il n'y a que les résultats de non unicité de [4] et [14]; pour tous les autres problèmes d'évolution l'unicité est une question ouverte. Concernant la régularité, lorsque $\varepsilon \neq 0$ rien ne semble connu pour les problèmes d'évolution et les résultats stationnaires sont des résultats sur la taille des singularités. Lorsque $\varepsilon=0$ il y a des estimations donnant de la régularité mais avec des constantes en double exponentielle de $t$; l'unicité autour d'une solution régulière repose sur une dépendance höldérienne des écarts en fonction des écarts entre les données avec un exposant qui se dégrade avec le temps. Si ces estimations sont précises, on n'est pas loin d'une régularité explosive.

On remarquera aussi que beaucoup de questions restent posées. Parmi celles-ci il y a l'étude du comportement des solutions lorsque $\varepsilon \rightarrow 0$, soit dans le problème d'évolution soit dans le problème stationnaire. De même l'étude du comportement asymptotique $t \rightarrow \infty$ n'a que des réponses partielles.

Un des enjeux est la compréhension mathématique des structures de parois brièvement mentionnées au début du paragraphe. 


\section{Quelques problèmes faisant intervenir l'énergie d'échange}

Un des premiers résultats mathématiques sur le ferromagnétisme concerne le problème de Cauchy. Il est dû à Visentin [31]. Voir aussi [10]. On considère le système d'équations

$$
\left\{\begin{aligned}
\partial_{t} E-\operatorname{curl} H & =0 \\
\partial_{t}(H+M)+\operatorname{curl} E & =0 \\
\partial_{t} M & =F\left(M, H+\varepsilon \mathcal{I}_{\Omega} \triangle M\right)
\end{aligned}\right.
$$

avec la condition de transmission (9) et des données de Cauchy en $t=0, E(0)=E_{0}, H(0)=$ $H_{0}, M(0)=M_{0}$, satisfaisant div $E_{0}=\operatorname{div}\left(H_{0}+M_{0}\right)=0, M_{0}$ vérifiant de plus

$$
\left|M_{0}(x)\right|=1 \text {, p.p. dans } \Omega,\left|M_{0}(x)\right|=0 \text {, p.p. dans } \mathbb{R}^{3} \backslash \bar{\Omega} \text {. }
$$

ThÉorème 2.1. On suppose $E_{0} \in L^{2}\left(\mathbb{R}^{3}\right), H_{0} \in L^{2}\left(\mathbb{R}^{3}\right)$ et $M_{0 \mid \Omega} \in H^{1}(\Omega)$. Alors il existe au moins une solution au problème de Cauchy pour (12), (9) qui vérifie $E, H \in$ $L^{\infty}\left(\left[0,+\infty\left[; L^{2}\left(\mathbb{R}^{3}\right)\right), M_{\mid[0,+\infty[\times \Omega} \in L^{\infty}\left(\left[0,+\infty\left[; H^{1}(\Omega)\right)\right.\right.\right.\right.$ avec en outre $\partial_{t} M_{\mid[0,+\infty[\times \Omega} \in$ $L^{2}([0, T] \times \Omega)$ et

$\left(\partial_{n} M \wedge M\right)_{\mid[0,+\infty[\times \partial \Omega}=0 \in L^{2}\left([0, T] ; H^{-\frac{1}{2}}(\partial \Omega)\right)$ pour tout $T>0$. De plus toute solution vérifie

$$
\partial_{t}|M(t, x)|=0, \text { p.p. dans } \mathbb{R}^{3}
$$

ainsi que

$$
\partial_{n} M_{\mid[0,+\infty[\times \partial \Omega}=0 \in L^{2}\left([0, T] ; H^{-\frac{1}{2}}(\partial \Omega)\right) .
$$

La propriété (15) résulte de (9) et de (13) qui entraine que $\left(\partial_{n} M \cdot M\right)_{\mid[0,+\infty[\times \partial \Omega}=0$. Les propriétés de régularité et d'unicité des solutions ne sont pas connues.

Concernant le comportement asymptotique $t \rightarrow \infty$, Carbou et Fabrie, avec les mêmes hypothèses que dans le théorème 2.1, montrent dans [10] le résultat suivant.

TÉorème 2. 2. Les éléments de l'ensemble $\omega$-limite d'une solution du problème de Cauchy précédent sont des solutions de l'équation stationnaire

$$
\left\{\begin{aligned}
\operatorname{curl} H & =0, \\
\operatorname{div}(H+M) & =0, \\
-\varepsilon \triangle M-\varepsilon M|\nabla M|^{2}-H+(M \cdot H) M & =0,
\end{aligned}\right.
$$

les deux premières équations étant posées au sens de $\mathcal{D}^{\prime}\left(\mathbb{R}^{3}\right)$, la troisième dans $\mathcal{D}^{\prime}(\Omega)$ avec la condition au bord (15), avec la condition supplémentaire (13) dans $\Omega$ pour $M$. Procédons

formellement en annulant les termes en $\partial_{t}$ dans (16) et dans $\Omega$. Notant $H_{\text {total }}=H+\varepsilon \triangle M$ on obtient $F\left(M, H_{\text {total }}\right)=0$. Tenant compte de l'expression de $F$, il vient

$$
H_{\text {total }} \wedge M=\beta M \wedge\left(H_{\text {total }} \wedge M\right) .
$$

$M$ étant non nul, les deux membres de l'égalité (17) sont orthogonaux sauf si $H_{\text {total }} \wedge M=0$. On a donc

$$
H+\varepsilon \triangle M=(H \cdot M) M+\varepsilon(\triangle M \cdot M) M .
$$

Prenant en compte la contrainte (13) dans $\Omega$, on vérifie que $(\triangle M \cdot M) M=|\nabla M|^{2} M$ ce qui donne l'expression de la dernière équation dans (16). 
Les équations (16) apparaissent comme les équations d'Euler de l'énergie totale définie comme la somme de l'énergie d'échange et de l'énergie démagnétisante

$$
\mathcal{E}(M)=\frac{1}{2} \int_{\Omega} \varepsilon|\nabla M|^{2}+\int_{\mathbb{R}^{3}}|H|^{2}=\frac{1}{2} \int_{\Omega}\left(\varepsilon|\nabla M|^{2}-M \cdot H\right),
$$

sous la contrainte $|M(x)|=1, x \in \Omega, H$ étant défini à partir de $M$ par les deux premières équations de (16) que l'on rappelle

$$
\operatorname{rot} H=0, \operatorname{div}(H+M)=0 .
$$

L'égalité entre les deux expressions à droite de (18) résulte d'un calcul direct que l'on peut faire en introduisant le potentiel $\Phi$ dont dérive $H=-\nabla \Phi$ et qui satisfait au sens des distributions dans $\mathbb{R}^{3}$

$$
\triangle \Phi=\operatorname{div} M .
$$

Il est naturel de rapprocher (18) de la fonctionnelle

$$
\mathcal{E}(M)=\frac{1}{2} \int_{\Omega}|\nabla M|^{2}
$$

dont l'équation d'Euler

$$
-\triangle M-M|\nabla M|^{2}=0
$$

définit, sous la contrainte $|M(x)|=1, x \in \Omega$, les applications harmoniques de $\Omega$ dans $S^{2}$, la sphère unité de $\mathbb{R}^{3}$. D'ailleurs on peut montrer que si $\varepsilon \rightarrow+\infty$ les points critiques de (18) convergent vers des points critiques de (20). On a donc une liaison avec un domaine très riche des mathématiques qui a fait l'objet de nombreux travaux ces dernières années. Le lecteur intéressé trouvera dans le livre de F. Hélein [16] un exposé contenant les derniers résultats dans le domaine des applications harmoniques. Cependant cette liaison que nous venons de mentionner a des limites. En particulier, l'application $M \mapsto H$ définie par (19) donne à la fonctionnelle (18) un caractère non local.

Carbou [9] a aussi démontré un théorème de stabilité lorsque la permittivité électrique tend vers 0 et montré la convergence vers des solutions des équations de Landau-Lifshitz dites quasi-stationnaires. L'approximation numérique de ces équations en 3-D par un schéma d'évolution en temps et des volumes finis en espace est entreprise par L. Halpern et S. Labbé [14] qui obtiennent aussi un résultat de non unicité pour l'équation quasi-stationnaire.

Reprenons le problème stationnaire (16). Comme pour les applications harmoniques un sujet central est l'étude de la régularité des points critiques de (18). On trouve dans [7] (Voir aussi [13], [6]) les résultats suivants

THÉorème 2.3. En dimension 2, toute solution de (16) est régulière dans $\Omega$.

THÉorÈme 2.4. En dimension 3, le support singulier de la solution de (16) qui réalise le minimum de (18) est formé de points isolés. Le support singulier d'une solution de (16) qui est un point critique stationnaire de (18) a une mesure de Hausdorff de dimension 1 nulle.

La situation est différente en dimension 2 et 3 comme dans le cas des applications harmoniques. La première partie du théorème 2.4 est à rapprocher d'un résultat de Schoen et Uhlenbeck [30] sur les singularités des applications harmoniques. Hardt et Kinderlehrer précisent dans [13], comme Brézis, Coron et Lieb [8] dans le cas harmonique, la forme de la singularité en $\frac{x}{|x|}$.

L'approximation numérique des solutions de (16) est délicate. On consultera [2] pour des énoncés précis, la description d' algorithmes performants (méthodes intégrales et géométries 3 -D) ainsi que [32], [1], [18]. 
Les résultats numériques montrent le comportement des solutions quand $\varepsilon \rightarrow 0$. La question sous-jacente est celle de la géométrie des parois dans un matériau ferromagnétique et du support singulier des solutions de

$$
\operatorname{div} M=0,|M(x)|=1, x \in \Omega, M(x)=0, x \in \mathbb{R}^{3} \backslash \bar{\Omega}, M \cdot n_{\mid \partial \Omega}=0 .
$$

Les questions mathématiques naturelles sont les suivantes:

1) Les solutions de (16) convergent-elles vers des solutions de (22)?

Le support singulier des solutions de (22) n'est pas arbitraire comme l'on remarqué depuis longtemps les physiciens. Voir dans [27] la section sur la méthode de Van den Berg pour les géométries 2-D. Cependant on peut montrer la chose suivante. Soit $\Omega \subset \mathbb{R}^{2}$ et soit $\mathcal{S}$ une structure arbitraire de parois qui compartimente $\Omega$. Alors il existe une solution de (22) dont le support singulier contient $\mathcal{S}$. Ces solutions ne sont pas dans $H^{1}\left(\Omega ; S^{1}\right)$. Supposons que la réponse à 1) soit affirmative. La question suivante est alors

2) Les limites de (16) effectuent-elles un tri des structures $\mathcal{S}$ ? La réponse est plus que probablement oui. Par conséquent la vraie question est la description du tri au moyen d'un principe variationnel prenant en compte la balance entre le cout d'une paroi en énergie d'échange et son coût en énergie démagnétisante.

\section{Stabilité du problème de Cauchy en l'absence d'éner- gie d'échange}

Le problème auquel on s'intéresse maintenant avec plus de détails est celui où $\varepsilon=0$ dans (12), c'est à dire lorsqu'il n'y a plus d'énergie d'échange. Le système d'équations devient

$$
\left\{\begin{aligned}
\partial_{t} E-\operatorname{curl} H & =0, \\
\partial_{t} H+\operatorname{curl} E & =-\partial_{t} M, \\
\partial_{t} M & =F(M, H) .
\end{aligned}\right.
$$

Il est posé dans l'espace tout entier. Le matériau magnétique apparait comme le support de $M$. Le modèle est discuté dans [21]. Le problème de Cauchy en dimension d'espace $d=1$ ainsi que d'autres propriétés (comportement asymptotique, approximation numérique ...) du modèle sont étudiés dans [21], [22].

Nous nous intéressons maintenant au problème de Cauchy dans $\mathbb{R}^{3}$ qui possède des propriétés de stablité. La fin du paragraphe présente des résultats qui ont été annoncés dans [19] et dont les démonstrations détaillées sont en cours de rédaction [20].

Précisons d'abord la notion de solution d'énergie finie.

Definition On dit que $U=(E, H, M)$ est une solution d'énergie finie dans $S_{T}=[0, T] \times$ $\mathbb{R}^{3}$ si les 3 composantes $E, H, M$ sont dans $C^{0}\left([0, T] ; L^{2}\left(\mathbb{R}^{3}\right)\right), M$ appartient à $L^{\infty}\left([0, T] \times \mathbb{R}^{3}\right)$ et si $U$ est une solution distribution de (23) telle que

$$
\operatorname{div} E=\operatorname{div}(H+M)=0 .
$$

Écrivant la seconde équation dans (23) comme $\partial_{t}(H+M)+\operatorname{curl} E=0$ on voit que

$$
\partial_{t}(\operatorname{div} E(t))=\partial_{t}(\operatorname{div}(H(t)+M(t)))=0
$$

de sorte que la condition sur la divergence se propage en temps. On obtient immédiatement les estimations de base sur $U$. D'abord comme

$$
F(m, h) \cdot m=0, m, h \in \mathbb{R}^{3}
$$


il en résulte, ainsi qu'on l'a déjà remarqué, que

$$
|M(t, x)|, \quad 0 \leq t \leq T
$$

reste constant au cours du temps. Ensuite Remplaçant $\partial_{t} M$ par $F(M, H)$ dans la seconde équation de (24) on voit que $(E, H)$ satisfait le système de Maxwell avec un second membre dans $L^{1}\left([0, T] ; L^{2}\left(\mathbb{R}^{3}\right)\right)$ car, par hypothèse, $M \in L^{\infty}$. Le champ électromagnétique $(E, H)$ vérifie donc l'estimation d'énergie habituelle. Comme

$$
F(m, h) \cdot h=\gamma \frac{\alpha}{1+\alpha^{2}} \frac{1}{|m|}|h \wedge m|^{2}, m, h \in \mathbb{R}^{3},
$$

il en résulte que

$$
\int_{\mathbb{R}^{3}}\left(|E(t, x)|^{2}+|H(t, x)|^{2}\right) d x, \quad 0 \leq t \leq T
$$

est une fonction décroissante du temps si $\alpha>0$ ou constante si $\alpha=0$ ce qui indique le caractère dissipatif ou conservatif du modèle. En résumé

$$
n_{0}(t)=\sqrt{\|E(t)\|_{2}^{2}+\|H(t)\|_{2}^{2}}+\|M(t)\|_{L^{2} \cap L^{\infty}} .
$$

vérifie

$$
n_{0}(t) \leq n_{0}(0), \quad 0 \leq t \leq T
$$

avec l'égalité si $\alpha=0$.

Dans le but de clarifier l'exposé des résultats mathématiques, on va tricher avec la physique et proposer deux jeux d'hypothèses sur le terme non linéaire qui different en régularité au passage par $M=0$.

HyPoTHÈSE 3.1. On suppose

$$
\begin{gathered}
F \in C^{\infty}\left(\mathbb{R}^{3} \times \mathbb{R}^{3} ; \mathbb{R}^{3}\right) \\
h \mapsto F(m, h) \text { is linear, } \quad m \in \mathbb{R}^{3} \\
F(m, h) \cdot m=0, \quad F(m, h) \cdot h \leq 0 \quad m, h \in \mathbb{R}^{3} .
\end{gathered}
$$

Lorsque $F$ vérifie l'hypothèse 1 il existe une fonction $C(R)$ telle que pour tout $R>0$,

$$
\left\{\begin{array}{rlrl}
\left|F\left(m^{\prime}, h\right)-F(m, h)\right| & \leq C(R)\left|m^{\prime}-m\right||h| & ,|m|,\left|m^{\prime}\right| \leq R \\
|F(m, h)| & \leq & R C(R)|h| & ,|m|,\left|m^{\prime}\right| \leq R
\end{array}\right.
$$

HYPothÈSE 3.1'. C'est l'hypothèse 3.1 sauf que (24) est remplaçé par

$$
F \in C^{\infty}\left(\mathbb{R}^{3} \backslash\{0\} \times \mathbb{R}^{3} ; \mathbb{R}^{3}\right)
$$

et les inégalités (27) sont supposées vérifiées pour tout $m, m^{\prime} \neq 0$.

La fonction

$$
F(m, h):=\frac{\gamma}{1+\alpha^{2}}\left(h \wedge m+\frac{\alpha}{\sqrt{\delta^{2}+|m|^{2}}}(m \wedge(h \wedge m))\right), \quad h, m \in \mathbb{R}^{3}, \delta>0
$$

satisfait l'hypothèse 3.1 tandis que la fonction définie en (4) qui est homogène de degré 1 en $m, h$ est seulement localement lipschitzienne et ne vérifie que l'hypothèse 3.1 '. 
Notons $\mathbf{U}_{\mathbf{0}}$ un sous-ensemble de $\left\{U_{0}=\left(E_{0}, H_{0}, M_{0}\right) \in L^{2}\left(\mathbf{R}^{3}\right)^{3} ; \operatorname{div} E_{0}=\operatorname{div}\left(H_{0}+\right.\right.$ $\left.\left.M_{0}\right)=0\right\}$ tel que $\mathbf{M}_{\mathbf{0}}=\left\{M_{0} ;\left(E_{0}, H_{0}, M_{0}\right) \in \mathbf{U}_{\mathbf{0}}\right\}$ soit dominé dans $L^{2}\left(\mathbf{R}^{3}\right) \cap L^{\infty}\left(\mathbf{R}^{3}\right)$.

Théorème 3.1. On suppose que $F$ vérifie l'hypothèse 3.1'. Alors l'ensemble U des solutions d'énergie finie $U$ sur $S_{\infty}$ qui vérifient $U_{\mid t=0} \in \mathbf{U}_{\mathbf{0}}$ est non vide. De plus si $\mathbf{U}_{\mathbf{0}}$ est compact dans $\left(L^{2}\left(\mathbf{R}^{3}\right)\right)^{3}$, alors pour tout $T>0, \mathbf{U}_{T}=\mathbf{U}_{\mid S_{T}}$ est compact dans $\left(C^{0}\left([0, T] ; L^{2}\left(\mathbf{R}^{3}\right)\right)\right)^{3}$.

THÉORÈme 3.2. On suppose que $F$ vérifie l'hypothèse 3.1'. Soit $U_{0} \in \mathbf{U}_{\mathbf{0}}$ tel que rot $E_{0}$ et rot $H_{0}$ appartiennent à $L^{2}\left(\mathbf{R}^{3}\right)$. Alors il existe une unique solution d'énergie finie sur $S_{\infty}$ vérifiant $U_{\mid t=0}=U_{0}$. En outre rot $E$ et rot $H$ appartiennent à $C^{0}\left(\left[0,+\infty\left[; L^{2}\left(\mathbf{R}^{3}\right)\right)\right.\right.$.

ThÉorème 3.3. On suppose que $F$ vérifie l'hypothèse 3.1. Si $U_{0} \in H^{2}\left(\mathbf{R}^{3}\right)$ alors l'unique solution d'énergie finie de données $U_{\mid t=0}=U_{0}$ appartient à $C^{0}\left(\left[0,+\infty\left[; H^{2}\left(\mathbf{R}^{3}\right)\right)\right.\right.$.

Pour tout $\lambda>1$ définissons $S_{\lambda}=\varphi\left(\lambda^{-1} D_{x}\right)$ où $\varphi \in C_{0}^{\infty}$ est une fonction de troncature, $0 \leq \varphi \leq 1$, portée par $|\xi| \leq 2$ et égale à 1 sur $|\xi|=1$. On s'intéresse à l'approximation du problème de Cauchy pour (23) définie par le système d'équations

$$
\left\{\begin{aligned}
\partial_{t} E^{\lambda}-\operatorname{curl} H^{\lambda} & =0 \\
\partial_{t} H^{\lambda}+\operatorname{curl} E^{\lambda} & =-S_{\lambda} F\left(M^{\lambda}, H^{\lambda}\right) \\
\partial_{t} M^{\lambda} & =F\left(M^{\lambda}, H^{\lambda}\right)
\end{aligned}\right.
$$

et les conditions initiales

$$
E_{0}^{\lambda}=S_{\lambda} E_{0}, H_{0}^{\lambda}=S_{\lambda} H_{0}, M_{0}^{\lambda}=M_{0} .
$$

THÉORÈme 3.4. On suppose que F vérifie l'hypothèse 3.1'. Soit $U_{0} \in \mathbf{U}_{\mathbf{0}}$. Alors pour tout $\lambda>1$, le problème de Cauchy (29)-(30) possède une unique solution globale $U^{\lambda}$ appartenant à $C^{1}\left(\left[0,+\infty\left[; L^{2} \times L^{2} \times L^{\infty}\right)\right.\right.$. De plus lorsque $\lambda \rightarrow+\infty, U^{\lambda}$ possède une sous-suite convergeant vers une solution d'énergie finie globale $U$ satisfaisant $U_{\mid t=0}=U_{0}=\left(E_{0}, H_{0}, M_{0}\right)$. Le premier

résultat établit l'existence de solutions d'énergie finie globales en temps, l'unicité restant une question ouverte. Il donne aussi une propriété de stabilité. Pour tout $T>0$, l'application qui, à une donnée initiale, associe une des solutions du problème de Cauchy est, d'une certaine façon, continue de $\mathbf{U}_{\mathbf{0}}$ muni de la topologie de $L^{2}$ dans $C^{0}\left([0, T] ; L^{2}\left(\mathbf{R}^{3}\right)\right)$.

Le second théorème démontre l'unicité du problème de Cauchy sous l'hypothèse que les données sont plus régulières: les champs $E$ et $H$ ont un rotationel dans $L^{2}$. Il dit aussi que cette régularité supplémentaire se propage.

Le dernier résultat montre que les solutions locales habituelles du problème de Cauchy à valeurs dans l'algèbre $H^{2}\left(\mathbf{R}^{3}\right)$ sont en fait globales en temps, de même que les solutions plus régulières.

Les preuves des théorèmes 3.2 et 3.3 utilisent une inégalité de Strichartz limite pour laquelle la dimension d'espace $d=3$ apparait comme critique. Ainsi les théorèmes 3.2 et 3.3 sont vrais pour $d \leq 3$ mais l'estimation principale sur laquelle ils reposent, qui concerne une équation des ondes semi-linéaire, n'est sans doute plus valable si $d \geq 4$. En revanche le théorème 3.1 ne nécessite aucune restriction sur la dimension d'espace sa démonstration utilisant des arguments très différents où la dimension n'intervient pas.

L'aspect critique de la dimension 3 se remarque sur la stabilité des estimations $L^{2}$ sur les rotationnels du champ électromagnétique. En dimension 2 les constantes dépendent de façon exponentielle du temps, alors qu'en dimension 3 la dépendance est en double exponentielle. Ces estimations sont-elles précises?

Les conclusions des théorèmes $3.1,3.2$ et 3.4 ne nécessitent que l'hypothèse 3.1 ' contrairement au Théorème 3.3. Mais il est utile dans ce cas de remplacer le théorème 3.3 par un autre 
résultat d'approximation fournie par une méthode de Galerkine ou une méthode de filtrage comme celle qui est décrite dans le Théorème 3.4 par exemple, et qui sont essentiellement équivalentes.

Les théorèmes $3.1,3.2$ ont un sens physique plus immédiat que le théorème 3.3 , à cause de l'hypothèse faite par les physiciens que $|M|$ est une constante non nulle dans $\Omega$ et nulle hors de $\Omega$.

\section{Une estimation a priori sur les rotationnels de $\mathbf{E}$ et $\mathbf{H}$}

On introduit la décomposition orthogonale habituelle de $L^{2}$

$$
L^{2}=L_{\|}^{2} \oplus L_{\perp}^{2},
$$

l'opérateur de projection $P_{\|}: L^{2} \rightarrow L_{\|}^{2}$ donnant la composante de rotationnel nul et $P_{\perp}$ : $L^{2} \rightarrow L_{\perp}^{2}$ celle dont la divergence est nulle. On utilisera la même notation pour les homologues de ces opérateurs agissant dans les espace $L^{p}, 1<p<+\infty$. Si $U$ est une solution d'énergie finie, sa composante $H$ n'est pas en général à divergence nulle. On a $H=H_{\perp}+H_{\|}$avec la relation $H_{\|}+M_{\|}=0$ : dans la partie de l'espace où $M_{0}(x) \neq 0$, qui figure l'emplacement du matériaux ferromagnétique, l'onde $H$ est, compte tenu de (3), la superposition de deux ondes non nulles se propageant respectivement aux vitesses 1 et 0 . Cette remarque sur les différents modes de propagation sera exploitée dans la preuve du Théorème 3.1.

Proposition 4.1 Soit $U$ une solution d'énergie finie suffisamment régulière sur $S_{T}$. Il existe alors une constante $C$ dépendant de $T$, de la norme $\|U(0)\|_{\mathcal{u}}$ et des normes de $\operatorname{rot} E(0)$ et rot $H(0)$ dans $L^{2}\left(\mathbf{R}^{3}\right)$, telle que

$$
\sup _{0 \leq t \leq T}\left(\|\operatorname{rot} E(t)\|_{2}+\|\operatorname{rot} H(t)\|_{2}\right) \leq C .
$$

On commence par remarquer que les normes $L^{2}$ des rotationnels de $E(t)$ et $H(t)$ sont contrôlées par celle du gradient complet de $H_{\perp}(t)$ avec des constantes ne dépendant que de la norme $\|U(0)\|_{\mathcal{U}}$. On constate ensuite que $H_{\perp}$, dont la norme dans $\left.C^{0}\left([0, T] ; L^{2}\right)\right)$ est contrôlée par $\|U(0)\|_{\mathcal{L}}$, satisfait une équation des ondes semi-linéaire dont le terme de source $g$ vérifie essentiellement

$$
\|g(t)\|_{2}=O\left(\left\|\left|H_{\perp}(t)\right|^{2}\right\|_{2}\right),
$$

avec des constantes ne dépendant que de la norme $\|U(0)\|_{\mathcal{L}}$.

Soit $u$ une fonction scalaire régulière qui satisfait sur $S_{T}$

$$
\square u=g, \quad\|g(t)\|_{2}=O\left(\left\|u(t)^{2}\right\|_{2}\right) .
$$

L'objectif est d'obtenir une estimation a priori sur l'énergie à l'instant $t, 0 \leq t \leq T$,

$$
n(t)=\sqrt{\frac{1}{2}\left\|\partial_{x} u(t)\right\|_{2}^{2}+\frac{1}{2}\left\|\partial_{t} u(t)\right\|_{2}^{2}} .
$$

en fonction de l'énergie $n(0)$ à $t=0$, avec des constantes ne dépendant que de la norme de $u$ dans $\left.C^{0}\left([0, T] ; L^{2}\right)\right)$. Lorsque $d=2$, l'inégalité de Sobolev $\|v\|_{2} \leq C\left\|\partial_{x} v\right\|_{1}$ appliquée à $v=u^{2}(t)$ permet de linéariser le carré dans (4) et d'obtenir immédiatement le résultat.

Pour $d=3$, la même stratégie, à base d'inégalités de Sobolev, ne permet qu'un contrôle local qui explose en temps fini. Il est aussi habituel dans ce type de problème d' utiliser des estimations $L^{q}\left(L^{p}\right)$ dans l'espace-temps. Si $p$ et $q$ sont des réels tels que

$$
\frac{1}{q}+\frac{3}{p}=\frac{1}{2}, \quad q>2,
$$


alors toute fonction régulière vérifie l’inégalité de Strichartz généralisée (cf. [12] par exemple)

$$
\|u\|_{L^{q}\left([0, T] ; L^{p}\left(\mathbf{R}^{3}\right)\right)} \leq C_{q, p, T}\left(n(0)+\|\square u\|_{L^{1}\left([0, T] ;\left(L^{2}\left(\mathbf{R}^{3}\right)\right)\right.}\right),
$$

Cependant l'estimation qui nous intéresse pour contrôler le terme $u^{2}$, qui correspond à $q=$ 2, $p=\infty$, est exclue. La constante $C_{q, p, T}$ explose comme un facteur de $p$ lorsque $p \rightarrow+\infty$; des contrexemples de Lindblad [26] et Klainerman et Machedon [23] indiquent qu'il est impossible d'espérer l'estimation pour $q=2$ et $p=+\infty$. La stratègie que nous présentons consiste cependant à suivre cette idée. Elle s'appuie sur une inégalité de Strichartz précisée en fréquence dans le cas limite $q=2$ et $p=+\infty$, dont un preuve est donnée dans [20].

Définissons pour $\lambda>1$ la famille de troncatures en fréquences $S_{\lambda}=\varphi\left(\lambda^{-1} D_{x}\right)$ où $\varphi \in C_{0}^{\infty}$ est portée par $|\xi| \leq 2$ et vaut 1 sur $|\xi|=1$.

LEMME Il existe une constante $c$ telle que pour tout $\lambda>0$, tout $T>0$ et toute fonction $u \in C^{0}\left(\left[0, \infty\left[; H^{2}\left(\mathbf{R}^{3}\right)\right)\right.\right.$,

$$
\left\|S_{\lambda}(u)\right\|_{L^{2}\left([0, T] ; L^{\infty}\left(\mathbf{R}^{3}\right)\right)} \leq c \sqrt{\log (1+\lambda T)}\left(n(0)+\|\square u\|_{L^{1}\left([0, T] ;\left(L^{2}\left(\mathbf{R}^{3}\right)\right)\right.}\right)
$$

Soit $u$ régulière sur $S_{T}$ satisfaisant (31). Posons $\alpha_{\lambda}(s)=\left\|S_{\lambda}(u)(s)\right\|_{\infty}, 0 \leq s \leq T$. D'après (31) il existe une constante $C$ qui ne dépend que de $\|u\|_{C^{0}\left([0, T] ; L^{2}\left(\mathbf{R}^{3}\right)\right)}$ telle que $\|\square u(s)\|_{2} \leq C\left\|u(s)^{2}\right\|_{2}$. On écrit $u^{2}(s)=u(s) S_{\lambda}(u(s))+u(s)\left(I-S_{\lambda}\right)(u(s))$ et on vérifie que

$$
\left\|u(s)^{2}\right\|_{2} \leq C\left(\alpha_{\lambda}(s)+\frac{n(s)^{2}}{\sqrt{\lambda}}\right)
$$

où $C$ ne dépend que de $\|u\|_{C^{0}\left([0, T] ; L^{2}\left(\mathbf{R}^{3}\right)\right)}$.

Soit $\left(t_{p}\right)_{p}$ une suite finie strictement croissante de réels, commençant avec $t_{0}=0$ et bornée supérieurement par $T$. Introduisons les quantités

$$
m\left(t_{p}, t_{p+1}\right)=\sup _{t_{p} \leq s \leq t_{p+1}} n(s)+\sup _{\substack{0 \leq \lambda \\ t_{p} \leq t \leq t_{p+1}}} \frac{\sqrt{\int_{t_{p}}^{t} \alpha_{\lambda}(s)^{2} d s}}{\left.c \sqrt{\log \left(1+\lambda\left(t-t_{p}\right)\right.}\right)}, \quad p \geq 0 .
$$

Le contrôle de $n(T)$ sera obtenu par le contrôle de la fonction $m$, lui-même obtenu en ajustant la croissance de la suite $\left(t_{p}\right)$ à la croissance de $m$ par une méthode de petits pas.

Additionnons l'estimation d'énergie pour $\square$,

$$
n\left(t_{p+1}\right) \leq n\left(t_{p}\right)+\int_{t_{p}}^{t_{p+1}}\|\square u(s)\|_{2} d s
$$

à celle que donne le lemme, appliqué entre $t_{p}$ et $t_{p+1}$. Il en résulte, compte tenu de (33), que

$$
m\left(t_{p}, t_{p+1}\right) \leq 2\left(n\left(t_{p}\right)+\int_{t_{p}}^{t_{p+1}}\|\square u(s)\|_{2} d s\right) .
$$

Reportant (32) dans (34), on obtient, en utilisant l'inégalité de Cauchy-Schwarz et la définition $(33)$ de $m(s, t)$, que pour tout $\lambda>0$,

$$
\begin{aligned}
& m\left(t_{p}, t_{p+1}\right) \leq 2 n\left(t_{p}\right)+ \\
& C \sqrt{\left(t_{p+1}-t_{p}\right) \log \left(1+\lambda\left(t_{p+1}-t_{p}\right)\right)} m\left(t_{p}, t_{p+1}\right)+C \int_{t_{p}}^{t_{p+1}} \frac{m\left(t_{p}, s\right)^{2}}{\sqrt{\lambda}} d s,
\end{aligned}
$$

ESAIM: Proc., VoL. 3, 1998, 85-99 
où $C$ ne dépend que de $\|u\|_{C^{0}\left([0, T] ; L^{2}\left(\mathbf{R}^{3}\right)\right)}$. Maintenant, grâce à (33) appliqué entre $t_{p-1}$ et $t_{p}$, on majore $n\left(t_{p}\right)$ par $m\left(t_{p-1}, t_{p}\right)$; ensuite on choisit $\lambda=m\left(t_{p}, t_{p+1}\right)^{2}$; enfin on majore l'intégrale dans (35) en utilisant la croissance de $m$. Pour simplifier, posons

$$
m_{0}=n(0), \quad m_{p}=m\left(t_{p-1}, t_{p}\right), \quad \delta_{p}=t_{p}-t_{p-1}, \quad p \geq 1 .
$$

Avec ces nouvelles notations et les majorations indiquées, l'inégalité (35) implique

$$
\frac{m_{p+1}-m_{p}}{m_{p}} \leq 1+C \sqrt{\delta_{p+1} \log \left(1+\delta_{p+1} m_{p+1}^{2}\right)} \frac{m_{p+1}}{m_{p}}+C \delta_{p+1} \frac{m_{p+1}}{m_{p}}, \quad p \geq 1
$$

Le choix

$$
\delta_{p}=\inf \left(\frac{1}{4 C}, \frac{1}{16 C^{2} \log \left(1+\frac{4 m_{p-1}^{2}}{C}\right)}\right), \quad p \geq 1
$$

assure que les deux derniers termes à droite de (36) sont inférieurs à 1 si l'on y remplace $m_{p+1}$ par $4 m_{p}$. Ceci entraine que, pour tout $s$ vérifiant $t_{p} \leq s \leq t_{p+1}$, on a $\frac{m\left(t_{p}, s\right)-m_{p}}{m_{p}}<3$ donc $\frac{m_{p+1}-m_{p}}{m_{p}} \leq 3$. Par récurrence, il en résulte que

$$
m_{p} \leq 4^{p} n(0), \quad p \geq 1
$$

Comme $\delta_{p}$ donné par (37) vérifie, à cause de (38),

$$
\delta_{p} \geq \frac{1}{32 C^{2} \log \left(1+2 \frac{4^{p-1} n(0)}{\sqrt{C}}\right)},
$$

le réel $t_{p}=\sum_{1 \leq j \leq p} \delta_{j}$ est minoré par $C_{1} \log p$, donc supérieur à $T$ pour $p$ supérieur à $e^{T / C_{1}}$, ce qui achève la preuve de la Proposition 4.1.

On remarque que $p \sim e^{T / C_{1}}$ et (38) donnent

$$
\sup _{0 \leq s \leq T} n(s) \leq 4^{e^{T / C_{1}}} n(0) .
$$

\section{Estimation $H^{2}\left(\mathbf{R}^{3}\right)$}

Proposition 5.1 Soit $T>0$ et $U$ une solution d'énergie finie sur $S_{T}$ suffisamment régulière. Il existe une constante $C$ ne dépendant que de $\|U(0)\|_{H^{2}}$ telle que

$$
\sup _{0 \leq t \leq T}\|U(t)\|_{H^{2}} \leq C \text {. }
$$

On écrit les équations pour les dérivées de $U$. Notant $L$ l'opérateur différentiel intervenant dans (24), on obtient

$$
\begin{gathered}
L U=O(|H|), \quad L\left(\partial_{x} U\right)=O\left(\left|H \| \partial_{x} M\right|\right), \\
L\left(\partial_{x}^{2} U\right)=O\left(\left|\partial_{x}^{2} M\right|,\left|\partial_{x} H\right|\left|\partial_{x} M\right|,|H|\left|\partial_{x}^{2} M\right|,\left|H \| \partial_{x} M\right|^{2}\right),
\end{gathered}
$$

où les constantes dans les $O$ ne dépendent que de la norme $\left\|M_{0}\right\|_{\infty}$.

Examinons l'équation pour $\partial_{x}^{2} U$, l'équation pour $\partial_{x} U$ se traitant de façon analogue. On a

$$
\left\|\left|H(t)\left\|\left.\partial_{x} M(t)\right|^{2}\right\|_{2}=O\left(\|\| H(t)\left\|\partial_{x}^{2} M(t)\right\|_{2}\right)\right.\right.
$$


la constante ne dépendant que de $\left\|M_{0}\right\|_{\infty}$. Posons

$$
n_{2}(t)=\sup _{0 \leq t \leq T}\left\|\partial_{x}^{2} U(t)\right\|_{2}
$$

On montre, en utilisant le même type d'argument qu'au paragraphe 4, que

$$
\left\|\left|\partial_{x} H(t)\right| \mid \partial_{x} M(t)\right\|_{2} \leq C\left(n_{2}(t)+\alpha_{\lambda}(t) n_{2}(t)+\frac{n_{2}(t)^{2}}{\sqrt{\lambda}}\right)
$$

et, en utilisant l'inégalité suivante du type Judovic,

$$
\left\|M_{\|}(t)\right\|_{\infty} \leq C\left(\left\|M_{0}\right\|_{L^{2} \cap L^{\infty}}\right) \log \left(\frac{\left\|\partial_{x}^{2} M(t)\right\|_{2}}{\left\|M_{0}\right\|_{\infty}}\right)
$$

que

$$
\left\||H(t)| \mid \partial_{x}^{2} M(t)\right\|_{2} \leq C\left(n_{2}(t) \log n_{2}(t)+\alpha_{\lambda}(t) n_{2}(t)+\frac{n_{2}(t)^{2}}{\sqrt{\lambda}}\right),
$$

où, dans (39) et (40), la constante $C$ ne dépendent que de $\left\|M_{0}\right\|_{L^{2} \cap L^{\infty}}$. L'estimation d'énergie pour le système symétrique $L$ donne

$$
n_{2}(t) \leq n_{2}(0)+C \int_{0}^{t}\left(\left(1+\alpha_{\lambda}(s)\right) n_{2}(s)+n_{2}(s) \log n_{2}(s)+\frac{n_{2}(s)^{2}}{\sqrt{\lambda}}\right) d s, 0 \leq t \leq T .
$$

Choisissons $\sqrt{\lambda}=n_{2}(T)$. L'inégalité précédente donne

$$
n_{2}(t) \leq n_{2}(0)+C \int_{0}^{t}\left(\left(2+\alpha_{\lambda}(s)\right) n_{2}(s)+n_{2}(s) \log n_{2}(s)\right) d s, 0 \leq t \leq T .
$$

Soit $n_{2}^{\sharp}(t)$ la solution de

$$
\frac{d}{d t} n_{2}^{\sharp}(t)=C\left(\left(2+\alpha_{\lambda}(s)\right) n_{2}^{\sharp}(s)+n_{2}^{\sharp}(s) \log n_{2}^{\sharp}(s)\right), 0 \leq t \leq T, n_{2}^{\sharp}(0)=n(0) .
$$

La fonction $n_{2}^{\sharp}(t)$ majore $n_{2}(t)$ pour $0 \leq t \leq T$ et satisfait

$$
\log n_{2}^{\sharp}(t) \leq e^{C t}\left(\log n_{2}(0)+C \sqrt{t} \sqrt{\int_{0}^{t} \alpha_{\lambda}(s)^{2} d s}+2 C t\right), 0 \leq t \leq T .
$$

En utilisant (33) et la fin de la démonstration de la Proposition 4.1, on majore

$$
\sqrt{\int_{0}^{t} \alpha_{\lambda}(s)^{2} d s}=\sqrt{\sum_{t_{p+1} \leq T} \int_{t_{p}}^{t_{p+1}} \alpha_{\lambda}(s)^{2} d s}
$$

$\operatorname{par} C(T, n(0)) \sqrt{\log \left(1+n_{2}(T) T\right)}$.

On déduit alors de (41), avec $t=T$, une majoration de $n_{2}(T)$ en fonction de $T, n(0)$ et $n_{2}(0)$.

La preuve du Théorème 3.3 est claire. Soit $U(0) \in H^{2}\left(\mathbf{R}^{3}\right)$. Supposons que le temps de vie $T$ de la solution $U$ du problème de Cauchy semi-linéaire (23) de données $U(0)$ soit fini. On sait qu'alors $\lim _{t \rightarrow T}\|U(t)\|_{\infty}=+\infty$ ce qui contredit la Proposition 4 qui affirme que $\lim _{t \rightarrow T}\|U(t)\|_{H^{2}}<+\infty$. La solution locale $U$ est donc globale. 


\section{$6 \quad$ Le Théorème 3.1 et la stabilité $L^{2}$}

Soient $\left(U^{n}\right)_{n}$ une suite de solutions d'énergie finie dans la bande $S_{T}$ telle que la suite $\left(U^{n}(0)\right)_{n}$ converge dans $L^{2}$ vers un élément qu'on note $U^{\infty}(0)$. A cause de l'estimation dans l'espace $\mathcal{U}$ on peut supposer que la suite $\left(U^{n}\right)_{n}$ converge faiblement dans $L^{\infty}\left([0, T] ; L^{2}\left(\mathbf{R}^{3}\right)\right)$ vers un élément noté $U^{\infty}$. L'objectif est de montrer que la convergence de $U^{n}$ vers $U^{\infty}$ a lieu dans $C^{0}\left([0, T] ; L^{2}\left(\mathbf{R}^{3}\right)\right)$. La clé est une estimation $L^{2}$ sur la différence $M^{p}(t)-M^{q}(t)$ en fonction de $M^{p}(0)-M^{q}(0)$. En écrivant

$$
\begin{aligned}
F\left(M^{p}, H^{p}\right)-F\left(M^{q}, H^{q}\right)=F\left(M^{p}, H^{\infty}\right) & -F\left(M^{q}, H^{\infty}\right) \\
& +F\left(M^{p}, H^{p}-H^{\infty}\right)-F\left(M^{q}, H^{q}-H^{\infty}\right),
\end{aligned}
$$

on constate que, si $\left|M_{0}^{n}(x)\right| \leq R$, on a, pour presque tout $(t, x)$,

$$
\begin{aligned}
\frac{1}{2} \partial_{t}\left|M^{p}-M^{q}\right|^{2} \leq C(R) \mid & H^{\infty}|| M^{p}-\left.M^{q}\right|^{2} \\
& +\left(F\left(M^{p}, H^{p}-H^{\infty}\right)-F\left(M^{q}, H^{q}-H^{\infty}\right)\right) \cdot\left(M^{p}-M^{q}\right) .
\end{aligned}
$$

Un poids ponctuel $e^{-2 a(t, x)}$ absorbe le premier terme du membre de droite si a est une primitive en $t$ de $C(R)\left|H^{\infty}(t, x)\right|$. Le choix précis

$$
a(t, x)=|x|^{2}+\int_{0}^{t} C(R)\left|H^{\infty}(s, x)\right| d s
$$

fournit une fonction a positive, définie et finie pour presque tout $x$ et pour tout $t$ et telle que $e^{-2 a(t, x)}$ appartient à tous les $L^{p}, 1 \leq p \leq \infty$. On a alors

$$
\begin{aligned}
& \frac{1}{2} \partial_{t}\left(e^{-2 a}\left|M^{p}-M^{q}\right|^{2}\right) \leq \\
& e^{-2 a}\left(F\left(M^{p}, H^{p}-H^{\infty}\right)-F\left(M^{q}, H^{q}-H^{\infty}\right)\right) \cdot\left(M^{p}-M^{q}\right) .
\end{aligned}
$$

Proposition 6.1 Il existe une constante $C(R, T)$ telle que pour tout $\delta>0$ il existe $N(\delta)$ tel que pour tout $p \geq N(\delta)$ et $q \geq N(\delta)$ et tout $t, 0 \leq t \leq T$,

$$
\left\|e^{-a(t)}\left(M^{p}(t)-M^{q}(t)\right)\right\|_{2} \leq C\left(\delta+\int_{0}^{t}\left\|e^{-a(s)}\left(M^{p}(s)-M^{\infty}(s)\right)\right\|_{2} d s\right)
$$

Passant à la limite $q \rightarrow \infty$ dans le membre de gauche de (17), on en déduit que la suite $M^{p}(t)-M^{\infty}(t)$ tend vers 0 dans l'espace $L^{2}\left(e^{-2 a(t, x)} d x\right)$. On en déduit le résultat final avec des arguments classiques.

La preuve de la Proposition 6.1 passe par l'examen du terme $F\left(M^{p}, H^{p}-H^{\infty}\right)$ dans (43), qu'on décompose en la somme

$$
F\left(M^{p}, H^{p}-H^{\infty}\right)=F\left(M^{p}, P_{\perp}\left(H^{p}-H^{\infty}\right)\right)+F\left(M^{p}, P_{\|}\left(H^{p}-H^{\infty}\right)\right) .
$$

L'intégrale en $t, x$ du premier terme à droite de (45) donne une contribution $\delta$, grâce à un argument de front d'onde une fois qu'on a remarqué qu'il s'agit du produit d'un terme en $M$ qui possède une dérivée par rapport à $t$ bornée dans $L^{2}$ et d'un terme $P_{\perp}\left(H^{p}-H^{\infty}\right)$ qui est borné dans $L^{2}$ avec $\square\left(P_{\perp}\left(H^{p}-H^{\infty}\right)\right)$ borné dans $H^{-1}$

L'autre terme $e^{-2 a(t)} F\left(M^{p}(t), P_{\|}\left(H^{p}(t)-H^{\infty}(t)\right)\right)=e^{-2 a(t)} F\left(M^{p}(t), P_{\|}\left(M^{\infty}(t)-M^{p}(t)\right)\right)$ s'écrit, après multiplication par $\left(M^{p}(t)-M^{q}(t)\right)$, comme la somme dans $L^{1}$

$$
\begin{aligned}
& F\left(M^{p}(t), P_{\|} e^{-a(t)}\left(M^{\infty}(t)-M^{p}(t)\right)\right) \cdot e^{-a(t)}\left(M^{p}(t)-M^{q}(t)\right) \\
& +F\left(M^{p}(t),\left[e^{-a(t)}, P_{\|}\right]\left(M^{\infty}(t)-M^{p}(t)\right) \cdot e^{-a(t)}\left(M^{p}(t)-M^{q}(t)\right),\right.
\end{aligned}
$$


où le crochet $[A, B]$ désigne le commutateur des deux opérateurs $A$ et $B$. La norme dans $L^{1}$ du premier terme se majore par une somme de carrés de normes $L^{2}$

$$
\begin{aligned}
\| F\left(M^{p}(t),\right. & \left.P_{\|} e^{-a(t)}\left(M^{\infty}(t)-M^{p}(t)\right)\right) \cdot e^{-a(t)}\left(M^{p}(t)-M^{q}(t)\right) \|_{1} \\
& \leq \frac{1}{2} R^{2} C(R)^{2}\left\|e^{-a(t)}\left(M^{\infty}(t)-M^{p}(t)\right)\right\|_{2}^{2}+\frac{1}{2}\left\|e^{-a(t)}\left(M^{p}(t)-M^{q}(t)\right)\right\|_{2}^{2} .
\end{aligned}
$$

Le deuxième terme de (46) donne une contribution $\delta$ car le commutateur $\left[e^{-a(t)}, P_{\|}\right]$est un opérateur compact sur les parties de $L^{2}$ dominées dans $L^{2} \cap L^{\infty}$ (on utilise (42)).

Sous l'hypothèse 3.1, la partie concernant l'existence de solutions globales $L^{2}$ du Théorème 3.1 peut maintenant se démontrer de la façon suivante. On approche les données initiales par régularisation par des unités approchées. Ces approximations forment un sous-ensemble $\mathbf{U}_{0}$ satisfaisant les propriétés données à la section 3 . Le théorème 3.3 associe aux données régulières des solutions globales du problème de Cauchy. La condition de stabilité $L^{2}$ du Théorème 3.1 achève la preuve.

\section{Références}

[1] M. AID, Simulation de la répartition en domaine des pièces polaires des têtes d enregistrement magnétiques couches minces, Thèse INPG, 22/02/1993.

[2] F. Alouges, Travail en cours.

[3] G. Anzelotti, S. Baldo \& A. Visintin, Asymptotic behaviour of the LandauLifshitz model of ferromagnetism, A ppl. Math. Optimization, 23, No. 3, (1991), 171-192.

[4] F. Alouges \& A. Soyeur, On global weak solutions for Landau-Lifshitz equations: existence and non uniqueness, - it Nonlinear Anal., Theory Methods Appl., 18, No. 11, (1992), 1071-1084.

[5] F. Bloch, Z. Phyzik, 74, (1932), 295.

[6] C. Bonjour, Inversion de systemes linéaires pour la simulation des matériaux ferromagnétiques. Singularités d'une configuration d'aimantation, Thèse UJF, 10/1996.

[7] G. Carbou, Regularity for critical points of a non local functional, Calculus of Variations, (à paraître).

[8] H. Brézis, J.-M. Coron \& E.H. Lieb, Harmonic maps with defects, Comm. Math. Phys., 107, (1986), 649-705.

[9] G. Carbou, Modèle quasi-stationnaire en micromagnétisme, Prépublication MAB, 1997.

[10] G. Carbou \& P. Fabrie, Comportement asymptotique des solutions faibles des équations de Landau-Lifshitz, Prépublication MAB, 1997.

[11] L.C. Evans, Partial regularity for stationnary harmonic maps into spheres, Arch Rat. Mech. Anal., 116, (1991), 101-163.

[12] J. Ginibre \& G. Velo, Generalized Strichartz inequalities for the wave equation, $J$. Funct. Anal., 133, n 1, (1995), 50-68.

[13] R. Hardt \& D. Kinderleher, Some Regularity Results in Ferromagnetism, (to appear).

[14] L. Halpern \& S. LabBé, Travail en cours.

[15] F. HÉLEIn, Régularité des applications faiblement harmoniques entre une surface et une variété riemannienne, -it C. R. Acad. Sci. Paris, série 1, 312, (1991), 591-596. 
[16] F. HÉlein, Applications harmoniques, lois de consevation et repères mobiles, Coll. Nouveaux essais, Diderot Editeur, Arts et Sciences, 1996.

[17] A. Hubert, Magnetic domains, à paraître.

[18] E.H. Khannous, Simulation du mouvement de parois et de lignes de Bloch dans les matériaux ferromagnétiques, Thèse UJF, 10/1996.

[19] J.-L. Joly, G. MÉtivier \& J. Rauch, Solutions globales du système de Maxwell dans un milieu ferro magnétique, Séminaire École Polytechnique, 1997.

[20] J.-L. Joly, G. Métivier \& J. Rauch, Article en préparation.

[21] P. Joly \& O. Vacus, Mathematical and numerical studies of 1D nonlinear ferromagnetic materials, Rapport INRIA, n 3024, 1996.

[22] P. Joly \& O. Vacus, Maxwell's equations in a 1D ferromagnetic medium: Existence and uniqueness of strong solutions, Rapport INRIA, n 3052, 1996.

[23] S. Klainerman \& M. Machedon, Space-time estimates for null-forms and the local existence theorem, Com. Pure Appl. Math., 46, (1993), 1221-1268.

[24] L. Landau \& E. Lifshitz, -it Physik A, Soviet Union 8, (1935), 153.

[25] L. Landau \& E. Lifshitz, Électrodunamique des milieux continus, Cours de physique théorique, t. 8, éditions Mir, Moscou, 1969.

[26] H. Lindilad, Counterexamples to local existence for semi-linear wave equation, - it Amer. J. Math. 118, n 1, (1996), 1-16.

[27] J. Miltat, Domains and domain walls in soft magnetic materials, mostly, Proceedings of the NATO ASI, Applied Magnetism, Erice 1992, R. Gerber, C.D. Wright and G. Asti eds. Kluwer Press.

[28] L. NÉEL, Cahiers Phys. 25, (1944), 1-20.

[29] R. Schoen, Analytic Aspects of the Harmonic Maps Problem, Math. Sci. Res. Inst. Publ., 2, Springer, (1984), 321-358.

[30] R. Schoen \& K. Uhlenbeck, A regularity theory for harmonic maps, J. Diff. Geom., 17, (1982), 307-335.

[31] A. Visintin, On Landau-Lifshitz equations for ferromagnetism, Japan J. of Appl. Math., 2, No. 1, (1985), 69-84.

[32] A. Vialdix \& Bagneres, Simulation de la structure de parois dans un matériau magnétique, Thèse INPG, 09/02/1990. 